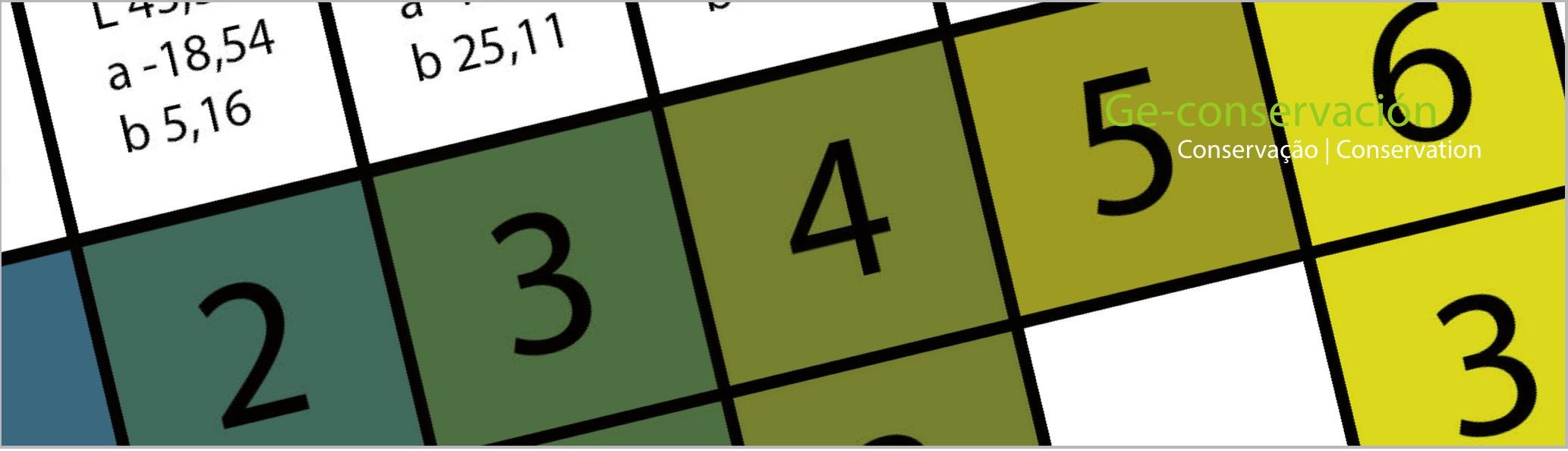

\title{
Los plásticos emisores de contaminantes gaseosos en las colecciones de Arte Contemporáneo. Propuesta de un protocolo para su conservación
}

\author{
Sara Liébana Molina
}

\begin{abstract}
Resumen: La conservación de las obras de arte realizadas en plásticos lleva siendo objeto de estudio desde los años noventa; sin embargo, se ha constatado tanto que la problemática de los plásticos emisores no ha sido estudiada en profundidad desde el campo de la conservación preventiva, como que no existen protocolos específicos para la conservación de este material. Este artículo muestra una propuesta de protocolo de conservación para obras de Arte Contemporáneo realizadas en plástico. Los resultados en los que está fundamentada han sido extraídos de una revisión bibliográfica en profundidad de las investigaciones especializadas y de un sondeo realizado a museos e instituciones con el fin de conocer qué pautas siguen en la actualidad para la conservación de este tipo de obras. Los resultados han sido complementados con datos obtenidos mediante un ensayo práctico que ha pretendido dar respuesta a las lagunas detectadas tras el análisis de los datos derivados de la revisión bibliográfica.
\end{abstract}

Palabras clave: plásticos, plásticos emisores, conservación preventiva, protocolo de conservación, Arte Contemporáneo

\section{Plastics emitters of gaseous pollutants in Contemporary Art Collections. Protocol proposal for its conservation}

Abstract: The conservation of plastic artworks has been considered a subject of study since the 1990s. However, it has been confirmed that the issue of emitter plastics has not been thoroughly studied with regard to preventive conservation. On top of that, it is known that there are no specific protocols for the preservation of this material. This paper presents a model protocol for the conservation of contemporary plastic artworks, as well as some fundamental results. These results were obtained during a thorough examination of specialized research, which was essentially a poll carried out in some museums and institutions. The purpose of this examination was to understand which are the current patterns followed for the conservation of this kind of artworks. Lastly, the results of a practical test are presented, aiming to give an answer to the gaps found after performing a data analysis of the bibliographical review.

Keywords: plastics, malignant plastics, preventive conservation, conservation protocol, contemporary art

\section{Os plásticos emissores de poluentes gasosos nas coleções de arte contemporânea. Proposta de um protocolo para sua conservação}

Resumo: A conservação das obras de arte realizadas em plásticos tem sido objeto de estudo desde os anos 90; no entanto, constatouse que a problemática dos plásticos emissores não foi estudada em profundidade no âmbito da conservação preventiva, e que não existem protocolos específicos para a conservação deste material. Este artigo apresenta uma proposta de protocolo de conservação para obras de arte contemporânea realizadas em plásticos. Os resultados em que está fundamentada foram extraídos de uma revisão bibliográfica em profundidade das investigações especializadas e de uma sondagem aos museus e às instituições, a fim de conhecer as orientações atuais para a conservação deste tipo de obras. Estes resultados foram complementados com dados obtidos através de um ensaio prático destinado a colmatar as lacunas identificadas na sequência da análise dos dados a revisão bibliográfica.

Palavras-chave: plásticos, plásticos emissores, conservação preventiva, protocolo de conservação, arte contemporânea 


\section{Introducción}

Desde que los artistas constructivistas como Naum Gabo o Antoine Pevsner incorporaron el plástico como material expresivo en sus obras de comienzos del siglo XX, este material no ha dejado de ser utilizado en el campo de la creación artística, de tal modo que su presencia en las colecciones de Arte Contemporáneo hoy día alcanza un elevado porcentaje.

Con apenas medio siglo de vida, estas primeras obras realizadas con plástico comenzaron a dar síntomas de inestabilidad, iniciándose así en los años noventa una línea de investigación que sigue vigente en la actualidad, debido a que la conservación de los plásticos constituye un desafío que crece en importancia a medida que los materiales van siendo amenazados por el paso del tiempo, ya que sus procesos de degradación son irreversibles y, en la mayoría de casos, imposibles de detener.

Un grave problema para la conservación de este material en las colecciones de arte, es la presencia de los plásticos emisores, es decir, aquellos que desprenden contaminantes gaseosos en todas o alguna de las fases de su vida útil; ya que pueden suponer un riesgo potencial tanto para los mismos plásticos como para otros materiales que se encuentren cercanos, desencadenando o acelerando sus procesos de degradación.

Si a esta realidad le añadimos que a día de hoy no existen datos fiables que nos permitan acometer intervenciones sobre las obras manteniendo su materia prístina; resulta fácil asumir que la mejor opción es la de desarrollar una serie de reglas que posibiliten preservarlas en las condiciones más óptimas con el objetivo de alargar su esperanza de vida.

\section{Objetivos y metodología}

El objetivo principal de la investigación que se recoge en este artículo es la elaboración de una propuesta de protocolo de conservación para obras de Arte Contemporáneo realizadas en plástico, haciendo especial hincapié en las estrategias para la preservación de los plásticos emisores.

Para alcanzar este objetivo, la metodología escogida se divide en tres grandes bloques:

Uno teórico, dedicado a la recopilación bibliográfica y documental, tanto de investigaciones generales de conservación de Arte Contemporáneo, como de las específicas de nuestro tema de estudio, que ha permitido comprobar que la materia elegida no ha sido abordada hasta ahora en profundidad desde una visión global.

Otro teórico-práctico, enfocado a la obtención de información mediante encuestas que incluye su diseño, envío, recogida y análisis gracias al cual se ha podido tanto detectar la ausencia de protocolos específicos para la conservación de obras de arte realizadas en plástico; como conocer las pautas y criterios que siguen actualmente los museos e instituciones para su preservación.

Tras la recopilación de la información obtenida en estos dos bloques, se efectuó una revisión crítica con el fin de comprobar la existencia de lagunas en relación con nuestro tema de investigación y así evaluar su interés, detectándose importantes vacíos.

Así, el tercer bloque, de carácter práctico, se dedicó al desarrollo de un ensayo empírico en el que se ha tratado de dar respuesta a las lagunas detectadas. Para ello, se ha seguido un planteamiento dividido en seis fases: diseño de un método propio para la realización del ensayo; preparación, envejecimiento y caracterización de muestras; preparación y montaje del sistema de medición; lectura, análisis e interpretación de datos obtenidos; aplicación de la metodología del ensayo práctico a un caso real; y análisis crítico y contrastivo de todos los resultados obtenidos.

\section{Plásticos emisores presentes en las colecciones de Arte Contemporáneo: qué emiten, a qué y quiénes afectan, y qué factores influyen}

El caucho, el nitrato de celulosa, el acetato de celulosa, el policloruro de vinilo (PVC), el poliuretano y el poliéster son los plásticos más señalados en las investigaciones especializadas como sospechosos de ser emisores de contaminantes gaseosos. Estos seis plásticos son además, junto al polimetilmetacrilato (PMMA), los que cuentan con mayor presencia en las colecciones de Arte Contemporáneo. Asimismo, la literatura sostiene que los contaminantes gaseosos más comúnmente emitidos son el dióxido de azufre, el dióxido de nitrógeno, y ácidos orgánicos e inorgánicos como el ácido acético, fórmico, adípico, nítrico, nitroso, clorhídrico, sulfúrico y el sulfuro de hidrógeno; siendo preciso señalar que estos compuestos volátiles son especialmente dañinos y se encuentran de modo más habitual en los entornos museísticos en su forma ácida (Grzywacz 2006).

En cuanto su repercusión en la estabilidad de otros materiales y la salud las personas, cabe señalar que Nazaroll y Cass (1991), Grzywacz (2006) y Sánchez Cabrero et al. (2015) afirman que si se respetan los umbrales aconsejados para la preservación a largo plazo de las colecciones patrimoniales, no existe ningún peligro para el personal expuesto; ya que los valores límite de contaminantes fijados para humanos están muy por encima de los aconsejados para la conservación de los Bienes Culturales. La revisión bibliográfica realizada también nos ha permitido concluir que algunos materiales como los metales, el vidrio, la cerámica, los tintes, los pigmentos, las pinturas, las piedras y los materiales celulósicos, fotográficos, de carbonato de calcio y proteicos son altamente sensibles a este tipo de emisiones, pudiendo sufrir daños que van desde la oxidación hasta la desintegración. 
También, cabe señalar que el modo de exponer o almacenar las obras realizadas en plásticos emisores influye directamente en su preservación a largo plazo, ya que el riesgo de acumulación de contaminantes gaseosos cuando las obras se hallan en espacios abiertos es prácticamente nulo; mientras que si se encuentran en el interior de contenedores estancos como vitrinas o cajas de viaje es muy elevado (Tétreault 2003; Sánchez Cabrero et al. 2015). De igual modo, dicho riesgo será mayor cuanto menor sea el volumen y la tasa de fuga de aire del contenedor; y mayor sea la temperatura, la humedad relativa y el tiempo que permanece la obra en su interior (Dahlin 2010; Tétreault 2019). Por otro lado, también es importante tener presente que la luz, el oxígeno, la temperatura y la humedad son los principales agentes de deterioro de los plásticos (Quye and Williamson 1999; Albus et al. 2007; Shashoua 2008; Waentig 2008; Lavédrine, Fournier and Martin 2012; Coughlin 2018); así como que los dos últimos además influyen de forma directa en su proceso de liberación de compuestos volátiles, ya que a medida que aumentan los grados y el porcentaje de humedad relativa, se produce un incremento en la tasa y en la velocidad de las emisiones (Hatchfield 2002; Coughlin 2018; Townsend, Hackney y Kearney 2019).

\section{Referentes para la elaboración de la propuesta de pro- tocolo de conservación}

- Situación actual; recomendaciones y pautas para la detección, el control y la conservación de los plásticos en las colecciones contemporáneas recogidas de investigaciones especializadas

Para la detección y el control de los compuestos volátiles ácidos emitidos por los plásticos, de la revisión bibliográfica se desprende que los dispositivos de lectura directa son la mejor opción a la hora de implementarlos en un protocolo de conservación debido a su facilidad de uso y su bajo coste; frente a los que requieren análisis de laboratorio, cuyos costes son mucho más elevados y precisan de científicos para interpretar los resultados. Entre los dispositivos de lectura directa se ha determinado que las AD Strips son la opción más óptima ya que, pese a que presentan algunas limitaciones, permiten detectar y controlar las emisiones derivadas de todos los plásticos en su forma ácida en tiempo real[ ${ }^{[1]}$, de forma económica, objetiva y sencilla.

En cuanto a las recomendaciones para la conservación de los plásticos que hacen los investigadores especializados, cabe señalar que proponen medidas encaminadas a eliminar o minimizar el riesgo de alteración o degradación; tanto generales, en las que se sugieren pautas de mantenimiento, identificación, almacenamiento y manipulación de carácter preventivo; como específicas, cuyo fin es mitigar o inhibir las situaciones de riesgo provocadas por sus principales agentes de deterioro extrínsecos, que se aplicarán únicamente cuando se detecta un riesgo derivado de alguno de estos parámetros. Dichas medidas serán detalladas más adelante.
- Situación actual; pautas de conservación generales y específicas recogidas mediante encuestas

Las respuestas obtenidas en las encuestas realizadas a los responsables de la preservación de quince colecciones contemporáneas (España: Artium, Centro Andaluz de Arte Contemporáneo, Centro Atlántico de Arte Moderno, Fundación Gala-Dalí, Museo Guggenheim de Bilbao y Vostell Malpartida; Europa: Die neue sammlung -The international design museum, Kunststoff-Museums-Verein e. V., Museum of Design in Plastics Statens Munseum for Kunts, Tampere Museum y Victoria \& Albert; América: Denver Art Museum, Museum of Modern Art y Pinacoteca do Estado de São Paolo) revelan que la medida de conservación que más se aplica es la de ajustar los parámetros ambientales al estado de conservación de la obra, especialmente la iluminación. También se detecta que, aunque la mayor parte de los encuestados asumen que las obras que presentan síntomas de degradación se deben separar de las que se encuentran en buen estado, esta medida no se pone en práctica en la mayoría de los casos por problemas de espacio o cuestiones curatoriales. Tampoco es común que se realicen de forma periódica, ni labores de mantenimiento, ni controles de contaminantes gaseosos; en el caso de los últimos se alega a la falta de recursos. Por último, en lo relativo a los criterios de actuación, cabe señalar que todos los participantes coinciden en que toman las decisiones de forma individualizada; si bien cada uno de ellos se apoya en directrices provenientes de instituciones muy diversas.

\section{— Resultados del estudio empírico}

En el ensayo práctico se testaron noventa muestras con forma de cubo de dos centímetros de lado, que conformaban tres sets de seis tipos de plástico, PMMA, poliéster, PVC, caucho, poliuretanoy acetato de celulosa (no se incluyó el nitrato de celulosa debido a que únicamente se localizaron proveedores que suministrasen este material en polvo), y cinco grados de fotoenvejecimiento cada uno (envejecimiento acelerado realizado en una cámara de luz xenón Solarbox 1500); que correspondían a 0 años, 25 años, 75 años, 150 años y 300 años si nos basamos en los factores de conversión utilizados por Thea Van Oosten en su libro PUR Facts (2011). El objetivo era dar respuesta a los interrogantes que se habían planteado tras la revisión bibliográfica, es decir, qué nivel de virulencia tiene cada plástico, la relación entre el grado de envejecimiento y la tasa de emisión, y la existente entre la concentración de contaminantes, el volumen del contenedor y el tiempo de exposición; así como conocer si el PMMA emana algún tipo de sustancia volátil nociva o no. Para ello, se introdujeron las muestras anteriormente descritas junto a una AD Strip en el interior de tres tipos de contenedor que emulaban las condiciones de obras embaladas $\left(90 \mathrm{~cm}^{3}\right)$, obras expuestas en vitrinas individuales $\left(320 \mathrm{~cm}^{3}\right)$ y obras expuestas en vitrinas compartidas $\left(1000 \mathrm{~cm}^{3}\right)$ durante tres periodos de tiempo diferentes: una semana, tres semanas y seis semanas. Para las lecturas de los resultados se 
utilizaron dos sistemas: comparación mediante patrón de equivalencias y espectrofotómetro.

Los resultados obtenidos han demostrado que en condiciones ambientales de museo $(60 \%+-2$ de humedad relativa y $19^{\circ} \mathrm{C}+-1$ de temperatura ambiental) el acetato de celulosa, el poliéster y el poliuretano son emisores; mientras que no se han detectado emisiones ácidas derivadas del caucho, el PVC y el PMMA. Los resultados obtenidos en el caso del caucho y del PVC discrepan de los hallados en la revisión bibliográfica, ya que la literatura señala a estos dos plásticos como emisores potenciales de ácido sulfúrico y sulfuro de hidrógeno, y ácido clorhídrico, respectivamente. Estas discrepancias se pueden deber en el caso del caucho, a que las emisiones ácidas derivadas de este plástico se producen en condiciones de humedad relativa alta (Quye y Williamson 1999), circunstancia que no se ha dado durante el ensayo práctico, en el cual este parámetro ha sido controlado en todo momento; mientras que en el caso del PVC, Tétreault (2019) afirma que no existen evidencias científicas de que este plástico emita ácido clorhídrico en condiciones de museo, siendo probable que las investigaciones que lo señalan como emisor potencial hayan obtenido estos datos de estudios realizados con fines industriales.

Asimismo, el ensayo práctico ha permitido clasificar los plásticos emisores en dos tipos: los que presentan una relación directa entre la tasa de emisión y el grado de envejecimiento, es decir, los que se vuelven más virulentos a medida que se fotooxidan, al que pertenece el poliuretano; y los que la presentan inversa, entre los que se encuentran el acetato de celulosa y el poliéster. Además, el análisis comparativo de los resultados obtenidos permite deducir que el poliéster es el plástico que registra las tasas de emisión más altas y, por tanto, el que presenta un grado de peligrosidad mayor; seguido del poliuretano y del acetato de celulosa.

Del mismo modo, los resultados obtenidos del ensayo práctico han probado la influencia del tiempo de exposición y el volumen del contenedor en el riesgo de acumulación de volátiles que se señalaban en la revisión bibliográfica. En el caso del primero, la relación es directa y está influenciada por la tasa de emisión del plástico, es decir, la concentración se incrementa cuanto más prolongado es el tiempo de exposición y más alta es la tasa de emisión; mientras que en del segundo es inversa, es decir, la concentración desciende de forma progresiva a medida que aumenta el volumen. También se ha constatado que el tiempo medio que tarda en pasar por cada nivel de concentración desde la ausencia hasta la concentración media es de cinco semanas en los contenedores pequeños y medianos, y ligeramente superior en los grandes; a partir de la concentración media este tiempo baja hasta las dos semanas.

Además, la experiencia obtenida durante su uso en el ensayo práctico, pone en evidencia que el lápiz de referencia proporcionado por el IPI junto a las AD Strips para realizar las lecturas presenta importantes carencias. A este problema, con motivo de esta investigación, se ha decido aportar una solución diseñando un patrón de equivalencias que corrigiese estas deficiencias; del mismo modo que se ha propuesto utilizar dicho patrón en combinación con un espectrofotómetro para obtener datos más objetivos y precisos, que nos permitan detectar cambios más sutiles [ver Figura 1].

\begin{tabular}{|c|c|c|c|c|c|c|c|}
\hline Coordenadas CIELAB $\left(\mathrm{L}^{*} \mathrm{a}^{*} \mathrm{~b}^{*}\right)$ & $\begin{array}{l}\mathrm{L} 41,63 \\
\mathrm{a}-10,19 \\
\mathrm{~b}-35,30\end{array}$ & $\begin{array}{l}\mathrm{L} 42,13 \\
\mathrm{a}-16,24 \\
\mathrm{~b}-15,35\end{array}$ & $\begin{array}{l}\mathrm{L} 43,38 \\
\mathrm{a}-18,54 \\
\text { b } 5,16\end{array}$ & $\begin{array}{l}\text { L } 45,16 \\
\text { a }-16,14 \\
\text { b } 25,11\end{array}$ & $\begin{array}{l}\text { L 54,34 } \\
\text { a -7,04 } \\
\text { b 45,21 }\end{array}$ & $\begin{array}{l}\mathrm{L} 65,18 \\
\mathrm{a}-74,24 \\
\text { b } 65,16\end{array}$ & $\begin{array}{l}\text { L } 85,22 \\
\text { a }-6,23 \\
\text { b } 85,36\end{array}$ \\
\hline \multicolumn{8}{|l|}{ Patrón de equivalencias propuesto } \\
\hline \multicolumn{8}{|l|}{ Patrón de equivalencias del IPI } \\
\hline Niveles de concentración & $\begin{array}{l}\text { Ausencia } \\
\text { acidez }\end{array}$ & $\begin{array}{c}\text { Concentración } \\
\text { baja }\end{array}$ & $\begin{array}{c}\text { Concentración } \\
\text { media - baja }\end{array}$ & $\begin{array}{c}\text { Concentración } \\
\text { media }\end{array}$ & $\begin{array}{c}\text { Concentración } \\
\text { media - alta }\end{array}$ & $\begin{array}{c}\text { Concentración } \\
\text { alta }\end{array}$ & $\begin{array}{c}\text { Concentración } \\
\text { muy alta }\end{array}$ \\
\hline Niveles de riesgo & & & & & & & \\
\hline
\end{tabular}

Figura 1.-Patrón de variación colorimétrica e interpretación de resultados para comparación con las lecturas de las A-D Strips. 
El método empleado en el ensayo práctico ha sido validado comparando los resultados obtenidos con los de un caso real, mostrándose gran concordancia entre los valores obtenidos y ninguna discrepancia; y cabe señalar que debido a su sencillez, eficacia y objetividad, el diseño del ensayo práctico utilizado es perfectamente aplicable a la propuesta de protocolo.

\section{Propuesta de protocolo}

Para la elaboración de la propuesta de protocolo de conservación para plásticos se han tenido en cuenta los aspectos analizados en la parte teórica y los datos extraídos de las encuestas y del estudio empírico que señalábamos en el punto anterior; así como la metodología recomendada por el Ministerio de Cultura y Deporte de España para la elaboración e implantación de planes de conservación preventiva (Ministerio de Cultura y Deporte 2019).

Este protocolo que ahora presentamos trata de cubrir por un lado, las lagunas detectadas;y por otro, de crear una herramienta de fácil aplicación, lo que permitiría generalizar su uso.

La estructura del protocolo se ha dividido en tres fases:

- Descripción de la obra y su entorno

- Detección y control de los agentes de deterioro

- Reducción e inhibición de los procesos de alteración y degradación

\section{- Descripción de la obra y su entorno}

Este apartado está destinado a conocer en profundidad tanto la obra, como el entorno en el que se encuentra, ya que sus características determinarán en gran medida las estrategias para su preservación. Por ello, se deben documentar fotográficamente y por escrito todos los aspectos que puedan influir de forma directa en su conservación.

En lo relativo a la obra es importante conocer su composición, caracterizando cada tipo de plástico y recogiendo la mayor información posible de los materiales de otra naturaleza; y su historia material, haciendo especial hincapié en la fecha de manufactura de los elementos plásticos; ya que como hemos visto el tipo de plástico y su grado de envejecimiento influyen directamente en su tasa de emisión.

La forma y dimensiones del material plástico también son determinantes, ya que dicha tasa será más elevada cuanto mayor sea el área de emisión, y por tanto, es esencial precisar la superficie $\left(e n \mathrm{~cm}^{2}\right.$ ) de cada uno de los elementos realizados en algún plástico emisor.

En cuanto a los elementos no plásticos, es importante identificar si la obra contiene alguno de los materiales conocidos por su sensibilidad a los compuestos volátiles que liberan los plásticos; es decir, metales, materiales celulósicos, materiales fotográficos, materiales de carbonato de calcio, vidrio, cerámica, materiales proteicos, tintes, pigmentos, pinturas o piedras.

También es de suma importancia conocer si la obra se encuentra en un espacio abierto, o si por el contrario está en un espacio cerrado de dimensiones limitadas ya que, por lo general, el riesgo de concentración de sustancias volátiles contaminantes en espacios de amplias dimensiones no es de relevancia. Sin embargo, como veíamos anteriormente, cuando se encuentra en un espacio confinado, como puede ser una vitrina o un embalaje, estas emisiones pueden acumularse convirtiéndose en una amenaza para su preservación.

En este sentido, cabe señalar que también existen diferencias entre los espacios cerrados en función de sus características. Los aspectos a tener en cuenta son el volumen del contenedor, la tasa de fuga de aire y con qué materiales está realizado; ya que el riesgo de acumulación de contaminantes gaseosos en un contenedor estanco será mayor cuanto menor sea el volumen y la tasa de fuga de aire del contenedor.

Asimismo, se debe controlar el tiempo de confinamiento de la obra dentro de su contenedor, puesto que cuando aumenta el tiempo de exposición, se incrementa la concentración de contaminantes. El mismo control se debe efectuar sobre las condiciones ambientales, ya que la humedad relativa y la temperatura son otros de los factores que influyen de forma directa, al producirse un incremento en la tasa de emisión y en la velocidad a la que se liberan los volátiles a medida que aumentan sus valores.

\section{— Detección y control de los agentes de deterioro}

Como ya anunciábamos, los principales agentes de deterioro son la luz, la temperatura, la humedad relativa, el oxígeno y los contaminantes emanados por los propios plásticos. Por lo que resulta imprescindible conocer cuáles son los niveles máximos y mínimos establecidos para estos parámetros ambientales.

\section{- Agentes de deterioro extrínsecos: temperatura, humedad re- lativa, iluminación y oxígeno}

Tras recopilary analizar en profundidad las recomendaciones realizadas por los investigadores especializados, se ha estimado que los niveles de temperatura más adecuados están comprendidos entre los $17-20^{\circ} \mathrm{C}$, mientras que los porcentajes de humedad relativa óptimos serán los que se encuentren entre el 45 y el $50 \%$. Además de mantenerse entre estos rangos, no hay que olvidar que estos parámetros tienen que permanecer lo más estables posibles, ya que su fluctuación también puede provocar daños.

En cuanto a la iluminación, teniendo en cuenta que la luz es uno de los principales factores degradantes de los plásticos, en este protocolo se ha optado por seguir la opción más 
restrictiva, es decir, no superar los 50 lux. Asimismo, sabiendo que el daño es acumulativo, se establece un límite anual de lux-hora/año para asegurarnos la correcta preservación de este tipo de obras, en concreto, 180.000 lux-hora/año. Del mismo modo, conviene asegurarse de que las fuentes de luz utilizadas no contengan radiación ultravioleta.

En el caso de que dichos parámetros ambientales no se encuentren dentro de los niveles recomendados, se debe pasar a la siguiente fase y adoptar las medidas pertinentes para la inhibición o reducción de sus efectos.

Por último, cabe señalar que si bien no se han encontrado investigaciones que determinen qué niveles de oxígeno son los más adecuados; de la revisión bibliográfica se desprende que la degradación de las obras se retrasa considerablemente en ausencia de oxígeno (The Plastics Historial Society 2015; Coughlin 2018). Pese a esto, si tenemos en cuenta que el oxígeno comprende el $21 \%$ de la atmósfera, se entiende que la preservación de los ítems de plástico en anoxia de forma permanente no es sostenible, y que únicamente se tomarán medidas para eliminar el oxígeno en situaciones de alto riesgo en las que la perdurabilidad de la obra dependa de ello.

- Agentes de deterioro intrínsecos: contaminantes emanados por los plásticos emisores

Para la detección y el control de los contaminantes emanados por los plásticos emisores vamos a utilizar dos métodos complementarios: la inspección visual y el muestreo de compuestos volátiles ácidos.

- Detección y control visual de signos de degradación en plásticos y materiales sensibles

Mediante la inspección visual podemos detectar y controlar si los propios plásticos y/o los materiales sensibles a sus emisiones muestran alguno de los síntomas característicos de su acción. Los síntomas más comunes, se recogen en la tabla que podemos observar a continuación. [Tabla 1]

\begin{tabular}{|l|l|}
\hline MATERIAL SENSIBLE & SÍNTOMAS MÁs COMUNES \\
\hline Metales & Deslustre y corrosión \\
\hline Materiales celulósicos & $\begin{array}{l}\text { Disminución de la resistencia, friabilidad, tinción de fibras y } \\
\text { desintegración }\end{array}$ \\
\hline Materiales fotográficos & $\begin{array}{l}\text { Oscurecimiento, descomposición, debilitamiento, } \\
\text { aceleración de los procesos de degradación, } \\
\text { empalidecimiento, reflejos plateados y foxing }\end{array}$ \\
\hline Materiales de carbonato de calcio & Eflorescencias \\
\hline Vidrio & $\begin{array}{l}\text { Pérdida de transparencia, pérdida de brillo, exudaciones y } \\
\text { craquelados }\end{array}$ \\
\hline Ccrámica & Eflorescencias \\
\hline Materiales proteicos & Craquelados, pulverulencia, red rot y pérdida de resistencia \\
\hline Tintes & Desvanecimiento \\
\hline Pigmentos & Oscurecimiento \\
\hline Pintura y piedras & Decoloración y erosión superficial \\
\hline Plásticos & $\begin{array}{l}\text { Oscurecimiento, amarilleamiento, decoloración, pérdida de } \\
\text { brillo, pérdida de transparencia, deformaciones, } \\
\text { eflorescencias, exudaciones, aumento de la porosidad, } \\
\text { aumento de la rigidez, aparicín de craquelados, fragilidad, } \\
\text { fisuras, agrietamientos, roturas, pulverulencia y } \\
\text { desintegración }\end{array}$ \\
\hline & \multicolumn{2}{|l}{} \\
\hline
\end{tabular}

Tabla 1.-Materiales sensibles a los contaminantes derivados de los plásticos emisores y síntomas más comunes.
- Muestreo de compuestos volátiles ácidos con AD Strips

Para el muestreo de compuestos volátiles ácidos con $A D$ Strips se deben tener en cuenta las recomendaciones de uso y manipulación publicadas tanto por el distribuidor, el Image Permanence Institute (2016), como por los investigadores especializados Nicholson y O'Loughlin (1996), Hatchfield (2002), Shashoua (2008) y Coughlin (2011).

En cuanto a las lecturas, se realizarán con un espectrofotómetro siguiendo las recomendaciones publicadas en el artículo Colourmeasurement of acid-detector strips for the quantification of volatile organic acids in storage conditions (Hackney 2016) y los datos se interpretarán con el patrón de equivalencias diseñado con motivo de esta investigación.

En este protocolo se van a tener en cuenta cinco tipos de muestreos, los cuales se pueden clasificar en dos grandes bloques: detección, que engloba el muestreo inicial y el de verificación; y control, al que pertenece el muestreo de seguimiento, el periódico y la monitorización temporal.

El muestreo inicial detecta si existe o no presencia de compuestos volátiles ácidos en el ambiente interior del contenedor en el que está expuesta o almacenada la obra. Para ello se introduce una AD Strip en su interior durante 24 horas; tras este periodo de exposición se realiza la lectura.

El muestreo de verificación determina si las emisiones detectadas en el muestreo inicial provienen de la obra o de los materiales con los que está realizado el contenedor. Para ello, se extrae la obra del contenedor en el que se encontraba, se aísla en uno nuevo de polietileno o vidrio (materiales que no interfieren en el análisis de volátiles), y se introduce una AD Strip en el interior del mismo. En este caso, lo que varía es que el tiempo de exposición es superior, la lectura se realizará tras una semana, tiempo mínimo cuando se introduce una obra en un contenedor limpio (Hackney 2016).

Asimismo, para asegurarnos de que la configuración del ensayo es adecuada, es imprescindible introducir una (contenedor vidrio) o dos (contenedor polietileno) tiras de control, en el interior de un contenedor vacío igual que en el que se ha metido la obra; y colocar otra en el exterior, junto a los contenedores con el fin de verificar que ni los contenedores, ni el ambiente exterior se encuentren contaminados.

El muestreo de seguimiento se realiza a obras que ya han sido muestreadas previamente dando negativo, con el fin de comprobar si su estado de conservación sigue siendo el mismo, o por el contrario ha variado. Los pasos para llevar a cabo este tipo de muestreo son los mismos que los del muestreo inicial.

El muestreo periódico está indicado para hacer un seguimiento de la concentración ácida del interior de los contenedores cerrados en los que se exhiben las obras 
realizadas en plásticos emisores, como por ejemplo vitrinas, con el objetivo de verificar que dicho ambiente no supone un riesgo para la correcta conservación de la obra. En este caso las mediciones se realizarán una vez por semana, aprovechando el día de cierre del museo, durante el tiempo que dure la exposición; suspendiendo una $A D$ Strip en el interior del contenedor, y realizando su lectura tras $24 \mathrm{~h}$ de exposición.

La monitorización temporal tiene como propósito controlar el ambiente interior de los contenedores cerrados en los que se almacenan las obras, con el fin de vigilar que no existe una acumulación de compuestos volátiles nocivos que pueda poner en riesgo la obra que contiene. La monitorización tiene una duración máxima de mes y medio, y las lecturas se realizan semanalmente. Para llevarla a cabo, se introduce una AD Strip en el interior del contenedor en el que está almacenada la obra y tras 24 horas de exposición se procede a efectuar la primera lectura, y se vuelve a introducir inmediatamente la tira en el contenedor. Las lecturas siguientes se realizarán semanalmente hasta alcanzar el mes y medio. Cabe destacar que es muy importante realizar las lecturas lo más rápido posible, a fin de que la tira permanezca poco tiempo fuera del contenedor para que no se produzca una pérdida de información.

- Interpretación de los resultados obtenidos mediante la inspección visual y el muestreo con AD Strips

Para la interpretación de los resultados obtenidos, se deben tener en cuenta los datos expuestos en el apartado correspondiente al ensayo práctico, en los que se determina que el tiempo medio que se tarda en pasar por cada nivel de concentración desde la ausencia hasta la concentración media es de cinco semanas en los contenedores pequeños y medianos, y ligeramente superior en los grandes; y que a partir de la concentración media este tiempo baja hasta las dos semanas.

Así como las interpretaciones publicadas en el manual de uso de las AD Strips, en las se advierte de que las emisiones pueden llegar a suponer un peligro significativo a partir de la concentración media, mientras que para llegar a afectar al personal expuesto se debería alcanza una concentración muy alta.

\section{- Muestreo inicial o muestreo de seguimiento}

Volátiles ácidos: no + signos de deterioro: no = inspección visual + muestreo de seguimiento en 3 meses.

Volátiles ácidos: no + signos de deterioro: sí = seguimiento semanal mediante inspección visual de los síntomas durante un mes. Si se detectan cambios, muestreo periódico o monitorización temporal; en caso contrario muestreo de seguimiento + inspección visual en 3 meses.
Concentración ácida media-baja o inferior = muestreo periódico o monitorización temporal.

Concentración ácida media o superior = implementación de medidas para la inhibición o reducción de contaminantes emitidos por los plásticos.

\section{- Muestreo de verificación}

Volátiles ácidos: no + signos de deterioro: no, se asume que la fuente emisora de acidez es el contenedor = sustituir el contenedor por uno que cumpla las recomendaciones para el almacenaje de este tipo de obras (ver el subpunto: contaminantes emitidos por los plásticos del apartado Reducción e inhibición de los procesos de alteración y degradación) + inspección visual + muestreo de seguimiento en 3 meses.

Volátiles ácidos: no + signos deterioro: sí = como en el caso anterior, sustituir el contenedor por uno que cumpla las recomendaciones para el almacenaje de este tipo de obras + seguimiento semanal mediante inspección visual de los síntomas durante un mes. Si se detectan cambios = muestreo periódico o monitorización temporal; en caso contrario = inspección visual + muestreo de seguimiento en 3 meses.

Concentración ácida media-baja o inferior = muestreo periódico o monitorización temporal.

Concentración ácida media o superior = implementación de medidas para la inhibición o reducción de contaminantes emitidos por los plásticos.

\section{- Muestreo periódico}

Concentración ácida media-baja o inferior + signos de deterioro: no = muestreo rutinario, es decir, una vez por semana.

Concentración ácida media-baja o inferior + signos de deterioro: sí = seguimiento diario mediante inspección visual. Si se detectan cambios = estudio individualizado en profundidad de la problemática de dicha obra y su entorno, debido a que existe la posibilidad de que algunos materiales sufran daños a concentraciones inferiores de las que son capaces de detectar las AD Strips; en caso contrario $=$ muestreo + inspección visual semanal hasta que finalice la exposición.

Concentración ácida media o superior = implementación de medidas para la inhibición o reducción de contaminantes emitidos por los plásticos.

\section{- Monitorización temporal}

La interpretación de los resultados de la monitorización 
temporal la vamos a dividir en dos bloques: los resultados de las lecturas intermedias y el resultado final, es decir, el obtenido tras mes y medio de exposición.

Lecturas intermedias: se debe proceder de la misma forma que en muestreo periódico.

\section{- Resultado final:}

Volátiles ácidos: no + signos de deterioro: no = inspección visual + muestreo de seguimiento en 3 meses.

Volátiles ácidos: no + signos de deterioro: sí = si se han detectado cambios = estudio individualizado como en el muestreo periódico; en caso contrario inspección visual + muestreo de seguimiento en 3 meses.

Concentración ácida baja: inspección visual + muestreo de seguimiento en 2 meses.

Concentración ácida media-baja: inspección visual + muestreo de seguimiento en 1 mes.

Concentración ácida media o superior = implementación de medidas para la inhibición o reducción de contaminantes emitidos por los plásticos.

\section{- Reducción e inhibición de los procesos de alteración y degradación}

A continuación se van a describir una serie de medidas encaminadas a eliminar o minimizar el riesgo de alteración o degradación de los plásticos emisores.

\section{- Mantenimiento, identificación, almacenamiento y manipulación}

Para la correcta conservación de las obras realizadas en plástico es muy importante tener en cuenta una serie de medidas preventivas que alargarán su vida útil.

Siempre que el estado de conservación o la naturaleza de la obra lo permitan, se recomienda limpiar la suciedad superficial regularmente mediante aspiración con ayuda de una brocha o con un tejido suave (gamuza de piel natural, gamuza o paño de microfibra (Lavédrine, Fournier y Martin 2012) que atrape el polvo. De forma excepcional se puede utilizar humedad, siempre y cuando esta acción se realice en el menor tiempo posible y acto seguido se seque la superficie con un paño seco, nunca con un secador u otro aparato que desprenda aire caliente; igualmente se desaconsejan en todo momento la utilización del resto de agentes de limpieza.

Para la identificación de las obras de este tipo se sugiere escribir los números de inventario con lápiz en cartones libres de ácidos que se depositarán junto a la obra; no siendo aceptable en ningún caso el uso de etiquetas autoadhesivas.

Asimismo, conviene especificar medidas concretas para las obras hinchables como que se deben almacenar siempre infladas y que el aire se debe insuflar mediante elementos mecánicos, nunca con la boca (Albus et al. 2007).

Por último, para la manipulación de los plásticos se recomienda utilizar guantes de nitrilo en todo momento (Coughlin 2018).

$\cdot \operatorname{Luz}$

Las obras realizadas en plástico deben estar expuestas el menor tiempo posible a la luz; para ello, se aconseja excluirla de las zonas de almacenamiento y limitarla durante la exhibición, iluminando las salas únicamente cuando haya público.

Asimismo, se recomienda iluminar siempre con fuentes lumínicas libres de radiación UV, con una iluminancia de 50 lux o inferior y no superando nunca los 180.000 lux-hora/ año.

\section{- Temperatura y humedad relativa}

En lo relativo a la temperatura y a la humedad relativa, como señalábamos anteriormente, lo más importante para evitar los daños que pueden causar estas dos magnitudes físicas es mantenerse sus niveles entre los $17-20^{\circ} \mathrm{C}$ y el $45-$ $50 \%$, respectivamente; así como evitar fluctuaciones. Para ello, es imprescindible que las obras se encuentren tanto en almacenaje como en exhibición en espacios con control climático.

En el caso de obras que tengan elementos plásticos de menos de un centímetro de espesor, que no estén ensamblados con otros materiales, que no presenten procesos de degradación activos y que además tengamos constancia de que va a permanecer en el almacén un largo periodo de tiempo, se puede recurrir al almacenaje en frío para reducir los índices de degradación química.

\section{- Oxígeno}

Para evitar los daños producidos por el oxígeno ambiental, se pueden exhibir o almacenar las obras en anoxia; siendo las atmósferas de nitrógeno y los absorbentes de oxígeno los dos métodos más utilizados para este fin. Si bien, estas medidas se tomarán únicamente en casos puntuales; ya que las atmósferas de nitrógeno, pese a dar buenos resultados, limitan notablemente el acceso a la obra y requieren muchos recursos, y los absorbentes de oxígeno están solamente recomendados en el caso de que el plástico ya haya perdido todo su plastificante. 


\section{- Contaminantes emitidos por los plásticos}

En cuanto a los contaminantes emitidos por los plásticos, existen dos medidas encaminadas a inhibir su acción: no almacenar ni exhibir las obras en contenedores cerrados, dado que propician el incremento de la contaminación gaseosa; y separar las obras que presentan signos de deterioro de las que se encuentran en buen estado de conservación con el fin de que las primeras no afecten a la estabilidad de las segundas.

Por otro lado, existen diferentes opciones para minimizar la acción de dichos contaminantes. La primera consiste en ventilar los espacios en los que se albergan las obras potencialmente emisoras con el fin de reducir la concentración de compuestos volátiles nocivos del aire; este sistema está indicado para todos los plásticos emisores, excepto el PVC, en el que puede inducir la pérdida de su plastificante. La segunda consiste en utilizar cartón de calidad archivo (que cumpla la norma ISO 11108:1996) para realizar los embalajes de las obras, ya que este material es capaz absorber los compuestos volátiles nocivos desprendidos por los plásticos emisores sin entrañar ningún riesgo para su estabilidad; el único aspecto que se debe tener en cuenta es que dichos embalajes se deben cambiar anualmente.

\section{Resumen de la información más relevante}

A continuación se exponene dos tablas-resumen en las que se recogen los plásticos emisores, los contaminantes emitidos, los materiales sensibles y los factores que influyen en el aumento del riesgo [tabla 2]; así como, los aspectos más relevantes de la propuesta de protocolo [Tabla 3].

\begin{tabular}{|l|l|l|}
\hline PLÁSTICOS POTENCIALMENTE EMISORES \\
\hline Caucho & Acetato de celulosa & Nitrato de celulosa \\
\hline Poliéster & Poliuretano & PVC \\
\hline CONTAMINANTES GASEOSOS MÁS COMUNMENTE EMITIDOS \\
\hline Dióxido de azufre & Ácido fórmico & Ácido clorhídrico \\
\hline Dióxido de nitrógeno & Ácido adípico & Ácido sulfúrico \\
\hline Sulfuro de hidrógeno & Ácido nítrico & \\
\hline Ácido acético & Ácido nitroso & \\
\hline MÁTERLALES SENSIBLES & Vidtio & Pigmentos \\
\hline Metales & Tintes & Materiales proteicos \\
\hline Cerámica & Piedras & Materiales de carbonato de \\
\hline Pinturas & Matcio \\
\hline Materiales celulósicos & FACTORES QUE INFLUYEN EN EL AUMENTO DEL RIESGO DE \\
\hline FACTCos \\
\hline GONCENTRACIÓN
\end{tabular}

Tabla 2.- Plásticos emisores, contaminantes emitidos, materiales sensibles y factores que influyen en el aumento del riesgo.

\begin{tabular}{|l|}
\hline OBRA: Composición, forma, dimensiones, área de emisión e historia material \\
\hline Elementos plásticos \\
\hline Tipo de plástico \\
\hline Fecha de manufactura \\
\hline Dimensiones \\
\hline Área de emisión (cm²) \\
\hline No plástico \\
\hline ¿Contiene algún material sensible? \\
\hline ENTORNO: Localización de la obra \\
\hline$\square$ Espacio abierto \\
\hline$\square$ Espacio cerrado \\
\hline Volumen del contenedor (cm ${ }^{3}$ ) \\
\hline AGENTES DE DETERIORO EXTRÍNSECOS: Temperatura, humedad y luz \\
\hline Umbrales seguros \\
\hline Temperatura: $17-20^{\circ} \mathrm{C}$ \\
\hline Humedad relativa: 45-50\% \\
\hline Iluminación: 50 lux \\
\hline AGENTES DE DETERIORO INTRÍNSECOS: contaminantes intrínsecos secundarios \\
\hline Inspección visual: detección de signos de degradación compatibles en plásticos y materiales \\
\hline sensibles \\
\hline Plásticos \\
\hline Materiales sensibles \\
\hline Muestreo de compuesto volátiles ácidos con A-D Strips: interpretación de resultados \\
\hline Muestreo inicial o muestreo de seguimiento \\
\hline Muestreo de verificación \\
\hline Muestreo periódico \\
\hline Monitorización temporal \\
\hline Umbrales seguros \\
\hline Materiales sensibles \\
\hline Personas \\
\hline MEDIDAS ENCAMINADAS AL LA ELIMINACIÓN O MINIMIZACIÓN DEL RIESGO \\
\hline DE ALTERACIÓN \\
\hline Mantenimiento, identificación, almacenamiento y manipulación \\
\hline Control de la iluminación \\
\hline Control de la temperatura y humedad relativa \\
\hline Eliminación o minimización del oxígeno \\
\hline Eliminación o minimización de los contaminantes emitidos por los plásticos \\
\hline
\end{tabular}

Tabla 3.- Resumen de los aspectos más relevantes de la propuesta de protocolo.

\section{Conclusiones}

En el desarrollo de esta investigación se ha concluido que es necesario un protocolo de conservación específico para plásticos debido a que los procesos de degradación de este material son irreversibles y prácticamente imposibles de detener; y que por tanto, la conservación preventiva es la opción más eficaz para asegurar su perdurabilidad.

De igual modo, se ha determinado que en dicho protocolo se deben tener muy en cuenta las características de la obra a preservary su entorno, debido a que algunos de los plásticos más presentes en las colecciones de Arte Contemporáneo (caucho, nitrato de celulosa, acetato de celulosa, PVC, poliuretano y poliéster) son emisores potenciales de contaminantes gaseosos, los cuales pueden provocar tanto la autocatálisis de los propios plásticos, como afectar a la estabilidad de otros materiales que se encuentren cercanos (metales, vidrio, cerámica, tintes, pigmentos, pinturas, piedras polímeros sintéticos o materiales celulósicos, fotográficos, de carbonato de calcio o proteico) o a la salud de las personas. 
En este sentido, también se debe tener presente que mediante el ensayo práctico se ha demostrado que en condiciones de museo el poliéster es el plástico más nocivo, seguido del poliuretano y el acetato de celulosa. Así como que el poliuretano se vuelve más virulento a medida que se fotooxida, mientras que con el poliéster y el acetato de celulosa ocurre justamente lo contrario. Asimismo, también se ha evidenciado que bajo estas condiciones el caucho, el PVC y el PMMA no emiten ningún tipo de volátil ácido; en este sentido cabe señalar que en el caso de los dos primeros parece que se debe a que las emisiones se producen en condiciones ambientales más extremas; mientras que en el del último se corrobora su inocuidad. Además se ha probado que el tamaño del contenedor en el que se expone la obra y el periodo de tiempo que permanece en su interior influyen directamente en el riesgo de acumulación de volátiles nocivos, ya que la concentración aumenta a medida que disminuye el cubicaje del contenedor y se incrementa el tiempo de exposición.

De la misma manera se ha llegado a la conclusión de que se debe destinar un apartado a la detección y control de los agentes de deterioro, en el se tenga en cuenta que la temperatura, la humedad relativa, la iluminación y el oxígeno son los principales agentes de degradación extrínsecos; y que para la correcta preservación de los plásticos sus niveles deben estar comprendidos entre los $17-20^{\circ} \mathrm{C}, 45-50 \%$ HR y 50 lux, para los tres primeros; mientras que en el caso del último, se ha determinado que la anoxia es la opción ideal, pero que únicamente se recomienda en casos puntuales debido a que presenta múltiples inconvenientes. En este punto cabe señalar además, que el aumento de la temperatura y la humedad relativa elevan el riesgo de concentración de volátiles.

También se ha concluido, que es de suma importancia detectar y controlar los volátiles desprendidos por los propios plásticos, habiéndose demostrado que las A-D Strips son la mejor opción para ello, al tratarse de un sistema eficaz, objetivo, económico y fácil de implementar; y que el umbral de riesgo para los materiales patrimoniales se sitúa en la concentración media, mientras que para las personas este se eleva hasta la concentración muy alta, debido a que son más resilientes a este tipo de contaminantes.

Por último, se ha determinado que se debe destinar un apartado a la reducción e inhibición de los procesos de alteración y degradación, en el que se debe tener en cuenta que tras analizar y contrastar las recomendaciones para la conservación de los plásticos que hacen los investigadores especializados con las respuestas obtenidas de los responsables de conservación de las colecciones contemporáneas encuestadas, se extrae que pese a que dichos profesionales conocen sobradamente las recomendaciones vigentes, únicamente se aplica de forma habitual la de ajustar los parámetros ambientales, especialmente la luz; mientras que el resto no suele llevarse a cabo principalmente por cuestiones curatoriales, problemas de espacio y/o falta de recursos.

\section{Notas}

[1] Una vez transcurrido el tiempo de exposición inicial recomendado que va desde las 24 horas en el caso de las obras que llevan tiempo dentro del mismo contenedor, y la semana en el caso de aquellas que se han introducido en un contenedor nuevo.

\section{Referencias}

ALBUS, S.; BONTEN, C.; KEßLER, K.; ROSSI, G. \& WESSEL, T. (2007). Plastic Art: A Precarious Success Story. Colonia: AXA Art Insurance Corporation.

COUGHLIN, M. (2011). "Monitoring Acidic Off-Gassing of Plastics", Conserve O Gram, 8/5: 1-5.

COUGHLIN, M. (2018). "Looking at Plastics. An introduction to caring for Plastics", Connecting to Collections Care. Washington D. C.: Foundation for advancement in conservation. https://www. connectingtocollections.org/caring-for-plastics/. [consulta: 27/11/2018]

DAHLIN, E. (2010). PROPAINT Improved Protection of Paintings during Exhibition, Storage and Transit. Norwegian Institute for Air Research

GRZYWACZ, C. M. (2006). Monitoring for Gaseous Pollutants in Museum Environments. Los Ángeles: Getty Conservation Institute.

HACKNEY, S. (2016). "Colour measurement of acid-detector strips for the quantification of volatile organic acids in storage conditions", Studies in Conservation, 61: 55-69. https://doi.org/10 $.1080 / 00393630.2016 .1140935$

HATCHFIELD, P. (2002). Pollutants in the museum environment. Practical Strategies for Problem Solving in Design, Exhibition and Storage. Londres: Archetype Publications

IMAGE PERMANENCE INSTITUTE. (2016). User's guide for A-D strips. Film base deterioration monitors. Nueva York: Rochester Institute of Technology

KENEGHAN, B., \& QUYE, A. (1999). "Degradation - Part 2: Degradation Causes". En: A. Quye \& C. Williamson (Eds.), Plastics Collecting and Conserving, National Museum of Scotland, 127.

LAVÉDRINE, B., FOURNIER, A., \& MARTIN, G. (Eds.). (2012). Preservation of Plastic Artefacts in Museum Collections. Francia: CTHS.

MINISTERIO DE CULTURA Y DEPORTE. (2019). Guía para la elaboración e implantación de planes de conservación preventiva. Madrid: Ministerio de cultura y deporte. 
NAZAROLL, W., \& CASS, G. (1991). "Protecting museum collections from soiling sue to the deposition of airborne particles", Atmospheric Environment, 25A: 841-852. https://doi. org/10.1016/0960-1686(91)90127-S

NICHOLSON, C., \& O'LOUGHLIN, E. (1996). “The Use of A-D Strips for Screening Conservation and Exhibit Materials", AIC. The Book and Papel Group Annual, 15.

OOSTEN, T. VAN. (2011). PUR Facts: conservation of polyurethane foam in Art and Design. Amsterdam: Amsterdam University Press.

SÁNCHEZ CABRERO, B., VILANOVA ANTA, O., CANELA, M. C., \& GÓMEZ ESPINOSA, T. (2015). "Calidad del aire interior de las vitrinas en el nuevo Museo Arqueológico Nacional", Boletín del Museo Arqueológico Nacional, 33: 367-382.

SHASHOUA, Y. (2008). Conservation of plastics. Materials, science, degradation and preservation. Oxford: $\mathrm{BH}$.

TÉTREAULT, J. (2003). Airborne Pollutants in Museums, Galleries and Archives: Risk Assessment, Control Strategies and Preservation Management. Canada: Canadian Conservation Institute.

TÉTREAULT, JEAN. (2019). "Products used in preventive conservation", CCI Technical Bulletins, 32.

THE PLASTICS HISTORIAL SOCIETY. (2015). "The Conservation of Plastics", Plastiquarian, 25. http://plastiquarian.com/?page id=14326. [consulta: 11/09/2019]

TOWNSEND, J. H., HACKNEY, S., \& KEARNEY, M. (2019). “A critical assessment of AD strips used in degradation studies for artworks made from cellulose acetate". En I. Soares \& P. Fuentez (Eds.), The Plastic Heritage Congress 2019: history, limits and possibilities, 29th - 31st May 2019, Lisbon Portugal.

WAENTIG, F. (2008). Plastics in Art: A Study from the Conservation Point of View. Colonia: Imhof Verlag.

\section{Autor/es}

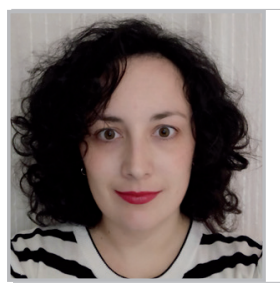

\section{Sara Liébana Molina}

sara.liebana@distritorestauracion.com

Distrito Restauración

https://orcid.org/0000-0002-4010-2226 restauradora y registro en diferentes empresas e instituciones como Distrito Restauración y el Museo Guggenheim de Bilbao.

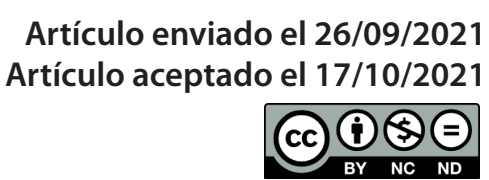

https://doi.org/10.37558/gec.v20i1.1061

Licenciada en Bellas Artes, Máster en Conservación y Exhibición de Arte Contemporáneo y Doctoranda del Programa Estrategias Científicas Interdisciplinarias en Patrimonio y Paisaje (ECIPP) de la Universidad del País Vasco con la tesis "Los plásticos emisores de contaminantes gaseosos en las colecciones de Arte Contemporáneo. Propuesta de un nuevo protocolo para su conservación". Colabora habitualmente como conservadora- 